\title{
La biblioteca escolar y su contribución a la motivación por la lectura
}

\author{
The school library and its contribution to the reading motivation
}

\author{
María del Carmen Hernández Valdés ${ }^{1}$ \\ Mireya Rodríguez Betancour ${ }^{2}$ \\ Leticia Echemendía Pérez ${ }^{3}$
}

\section{Resumen}

La práctica de lecturas rutinarias y tradicionales durante el proceso educativo se ha convertido en una problemática, sobre todo, cuando el escolar necesita sentir esta actividad como disfrute y creación personal. En el artículo se identifican problemas latentes al respecto y se plantean las bases teóricas teniendo en cuenta aportes de diferentes ciencias y disciplinas afines a la didáctica de la lectura que son coherentes con las ideas educativas que identifican la tradición pedagógica cubana. Siendo el principal objetivo, exponer las tendencias y los principales presupuestos asumidos que sustentan el problema relacionado con la motivación por la lectura. Las actividades proyectan el proceso de formación de lectores de un modo dinámico y creativo, a partir de una perspectiva que integra el empleo de técnicas de animación de la lectura, un estilo participativo y democrático, provocando efectos emotivos en los escolares. El estudio se desarrolló en el período de septiembre de 2018 a marzo de 2019. Para evaluar la efectividad del estudio se empleó el método de experimento pedagógico, en su modalidad de pre - experimento, con un diseño de pre - test y pos - test. Los efectos positivos logrados en los escolares del grupo experimental con respecto a los niveles de motivación, han demostrado la pertinencia y efectividad de las actividades propuestas.

Palabras clave: Actividades de animación; lectura; proceso de formación de lectores; motivación.

\section{Abstract}

The practice of routine and traditional readings during the educational process has become a problem, especially when the scholar needs to feel this activity as enjoyment and personal creation. In the article latent problems are identified and the theoretical

1 Master en Ciencias de la Educación. Profesora y coordinadora de la Carrera Licenciatura en Educación Primaria de la Universidad “José Martí Pérez" Sancti Spíritus. Correo: mchernandez@uniss.edu.cu ORCID: https://orcid.org/oooo-ooo1-5580-9833

2 Master en Ciencias de la Educación. Profesora de la Universidad “José Martí Pérez” Sancti Spíritus. Correo: mireyarb@uniss.edu. cu ORCID:https://orcid.org/oooo-0001-5232-0151

3 Licenciada en Ciencias Exactas. Profesora y coordinadora de la Carrera Ingeniería Agrónoma de la Universidad "José Martí Pérez" Sancti Spíritus. Correo: leticiaea@uniss.edu.cu. Cuba ORCID: https://orcid.org/oooo-ooo3-0158-9112

Recibido: 23/04/2019 Aprobado: 30/04/2019 
bases are considered taking into account contributions from different sciences and disciplines related to the didactics of reading that are coherent with the educational ideas that identify the Cuban pedagogical tradition. Being the main objective to expose the tendencies and the main assumed assumptions that sustain the problem related to the motivation for reading. The activities project the process of training readers in a dynamic and creative way, from a perspective that integrates the use of reading animation techniques, a participatory and democratic style, causing emotional effects in school children. The study was carried out from September 2018 to March 2019. To evaluate the effectiveness of the study, the pedagogical experiment method was used, in its pre-experiment modality, with a pre-test and post-test design. The positive effects achieved in schoolchildren of the experimental group with respect to motivation levels have demonstrated the relevance and effectiveness of the proposed activities.

Keywords: Animation Activities; Reading; Reader Training Process; Motivation.

\section{Introducción}

El interminable y siempre enardecido debate en torno a la motivación de la lectura, sirve para mantener a los bibliotecarios y otros promotores conscientes de que esta constituye una vía indispensable para conservar la lectura como uno de los elementos imprescindibles de la vida intelectual, emocional, cultural y social de las personas; razones suficientes para justificar el lugar prioritario que ocupa en el plan de estudio de la escuela primaria y en particular en el currículo bibliotecario.

Los cambios operados en los currículos y en la organización escolar, junto a los diferentes Programas de la Revolución y el Programa Nacional por la Lectura que hoy se desarrollan en la escuela, implican una transformación en la actividad docente educativa, a la vez que demandan de la biblioteca escolar un papel más activo y protagónico en este proceso, en virtud de elevar el nivel de motivación de los escolares por la lectura.

En este sentido, juega un papel fundamental el proceso de formación de lectores que tiene lugar en el ámbito pedagógico, donde la biblioteca realiza una labor vinculada al maestro que interactúa desde los primeros grados de la escuela, para que estos sientan interés y desarrollen el gusto por la lectura como una opción para su disfrute personal.

La Revolución Cubana ha hecho del libro un patrimonio real de todos, extendiendo a todo el país la Feria internacional de libro, pero aún no se lee lo suficiente, ni de la manera más lúcida y provechosa. Todavía no se educa a los escolares, desde las edades más tempranas, en el amor al libro. Por tales razones nos corresponde a los educadores, con el inmenso poder del ejemplo, compensar esas carencias y convertir 


\section{EDUCACIÓN}

todos los centros escolares en sitios donde se reverencie la lectura y se considere la formación de lectores como una de las tareas cardinales de la labor educativa.

De gran valor para el tema que se aborda son los aportes de diferentes estudiosos de la temática. El problema de la motivación por la lectura ha sido objeto de análisis, reflexión y tratamiento de diversos pedagogos, entre los que se encuentran los aportes realizados por investigadores cubanos Henríquez Ureña, (1975), Fowler Calzada, (2000), Arias Leiva, (2003, 2008, 2010) y Rodríguez Pérez, (2003), todos coinciden en la necesidad de la lectura, que debe realizarse de forma consciente, como un acto de placer y ejercicio intelectual y realizarla en momentos de ocio que se busca y se disfruta.

Dentro de las investigaciones sobre la lectura, es sin duda, la motivación de los escolares una de las más prolíferas, ya sea desde la labor de los maestros como desde la bibliotecaria, así se pudo comprobar en informaciones ofrecidas en nuestro territorio por: Herrera Rojas, (2009) y García González, (2011).

No obstante al aporte significativo dado por estos estudiosos, la motivación de los escolares por la lectura, sigue siendo una problemática no resuelta, permitiendo estudios ajustados a otros contextos. Tal es el caso de la investigación que se presenta en la que se profundiza en el trabajo con este proceso, cuya motivación elemental ha estado condicionada por las limitaciones constatadas por las autoras, que apuntan a la presencia de las manifestaciones que siguen:

1. Predisposición y/o indiferencia durante el acto lector.

2. Preferencias por los libros sencillos, de fácil comprensión y con predominio de ilustraciones.

3. No asocian la lectura como un acto placentero, sino como una obligación realizada bajo presión del docente.

4. Asocian el leer a una mera rutina escolar más, a un simple mecanismo de instrucción que se practica por cumplir con tareas escolares.

5. Muy pocos solicitan préstamos de libros en la biblioteca.

6. Limitado número de escolares practican la lectura recreativa en el tiempo libre.

7. Es insuficiente la participación en actividades relacionadas con el proceso lector.

En virtud de compensar esas necesidades se determina como objetivo: Aplicar actividades de animación de la lectura que contribuyan a la motivación por la lectura en los escolares de sexto grado de la escuela primaria "José Martí".

El aporte práctico está dado por las actividades de animación de la lectura dirigidas a la motivación de la lectura, su concepción y aplicación consecuente permite en los escolares que la lectura, además de una necesidad, llegue a constituir un placer ineludible. Como elemento novedoso se considera la forma en que se presentan las 
actividades de animación de la lectura, de manera que propicien un modo de integración con un estilo participativo y democrático; con un empleo de técnicas de animación de la lectura, en función de provocar efectos emotivos que hagan vivir al escolar la acción.

\section{Revisión de literatura}

La reorientación didáctica en el campo de la enseñanza de la lectura, deriva del supuesto central de la recepción del lector e implica analizar los factores y elementos condicionantes que intervienen en todo el proceso de lectura. El enfoque actual centra su interés, sobre todo, en potenciar la implicación personal seguida durante el proceso de lectura y redunda en la concepción de acciones en función de desarrollar y estimular la lectura.

La lectura representa una de las victorias arduas y sublimes de la humanidad. Se reconoce como un factor de marcada relevancia en la civilización ligada con su desarrollo intelectual, espiritual y social. Su historia, tanto en Cuba como en el mundo, está signada por connotados acontecimientos de distinta naturaleza (científicos, tecnológicos, sociales, culturales, educativos) que en sus mutuas relaciones trazan todo su trayecto cronológico y evolución ideológica; es una de las habilidades fundamentales destinadas a la obtención de información.

La buena lectura, comprende no sólo el conocimiento del significado literal de un texto, es preciso descifrar lo que se infieren del talento del autor, de su tono, de su intencionalidad y su actitud hacia el tema que trata, hacia sus lectores y hacia sí mismo, por lo que modifica el pensamiento y la conducta (Fowler, 2000, p. 13).

Herrera, R.L. (2009; p. 18) plantea: “(..) es un espacio para el vuelo imaginativo, para la audacia de pensamiento, para una jubilosa libertad, tanto por la elección de los textos como por la recreación que en la mente de cada ser humano los realiza y completa, y que los haga nacer a la vida y renovarse sin cesar." Este concepto se asume en la investigación, por considerarlo uno de los más completos; pues ve la lectura como la forma mediante la cual se adquieren nuevos conocimientos, que pueden ser transmitidos desde los textos de cada grado hasta llegar a aquel que por necesidad o curiosidad se acude y cada persona puede interpretar desde su punto de vista, además mediante la lectura se viaja a otros mundos, se conocen a los otros y a nosotros mismos, y se viven aventuras apasionantes en diferentes situaciones. Esta posee un enorme poder de satisfacción porque leer es encontrarse con otra forma de vivir y sentir. Con la lectura comienza otra vida.

El especialista, refiere que los educadores han de esforzarse por saber a fondo qué les gustaría leer a los niños, no sólo como tendencia colectiva de determinado grupo de edad, sino a nivel de desarrollo de sus actividades lectoras y de sus capacidades psíquicas. Cuando las dificultades en el dominio en el aspecto técnico de la lectura 


\section{EDUCACIÓN}

resultan un obstáculo, es natural que el lector se aleje del libro, en un mundo en que las imágenes del cine, la televisión y el video les proporciona un placer que implica un mínimo de esfuerzo (Herrera, 2009).

La lectura sirve para encontrar la posibilidad de fantasear, para enriquecer el universo con sus posibilidades recreativas y potenciar la capacidad de ocasionar ideas nuevas, integradas a la experiencia personal, lo que constituye su función recreativa, que no significa que el gusto de leer anule el aprendizaje, sino que este sea un factor que desarrolla el conocimiento.

Es indudable, pues, que la actitud del lector ante el libro es de gran importancia en la lectura. El lector común, es decir, el que no es ni crítico profesional, ni erudito, ni artista literario, es un personaje importante; porque la lectura no es un proceso pasivo, sino eminentemente activo, si se realiza como es debido.

El lector común, el simple aficionado, lee por placer personal, para obtener cierta experiencia. Su inclinación lo guía a organizar y guardar en su acervo espiritual un todo coherente, sacando de la obra que lee una figura humana, el cuadro de una época, o una pura emoción expresada en sonidos y ritmos ¿Cómo se debe leer un libro? En materia de lectura a nadie se le puede dar normas absolutas; sólo se pueden ofrecer ideas y sugestiones. Esto debe ser así, porque si se quiere que la lectura sea fructífera se debe respetar en el lector la libertad de apreciación. Cada lector debe llegar por sí mismo a sus propias conclusiones (Henríquez, 1975, p. 21).

El buen lector aspira a comprender, para lograrlo deja a un lado, al empezar, sus opiniones y perjuicios y tratar de seguir al autor cuya obra lee; no de dictarle lo que debe decir, sino de identificarse con el libro. Si por el contrario, el lector resiste, se enfrenta a él haciendo reservas mentales y en actitud de crítica destructiva al empezar, no sacará provecho alguno de lo que lee. Si se abre su mente lo más posible, los matices y los detalles que por ser muy finos le podrían pasar inadvertidos, lo llevará poco a poco a sentir la esencia de un vivir humano que no será igual a ningún otro, y comenzará darse cuenta de lo que el autor está tratando de decirle.

Proyectar el trabajo en este sentido se traduce en un proceso de formación de lectores, es decir, en modos de utilización, de comprensión, de apropiación y de gozo por los textos. Esta es una tarea ardua, de mucho tiempo, que exige un marco de cooperación de todos aquellos que promueven la lectura. A consideración de Herrera (2009): La formación de un lector es una tarea difícil, por diversas razones de orden sociocultural, psicológico y pedagógico. Tomar conciencia de este hecho es decisivo para una labor de promoción eficaz (p. 8). 
García González (2011), afirma: "La formación de lectores será más efectiva si en lugar de la improvisación y el espontaneísmo partimos de concepciones científicas, que se han acumulado como un valioso caudal de experiencias realizadas por educadores del mundo entero, recogidas en una bibliografía cada vez más numerosa" (p.12).

Ante estas realidades la escuela no debe, no puede seguir parapetada en una concepción de lectura y en prácticas de lecturas rutinarias y tradicionales; necesita replantearse todo su hacer, y en este hacer, la lectura sigue siendo aprendizaje básico que se debe atender, renovar, reconceptualizar y transformar. Se debe sentir que la lectura es una de las formas de aprendizaje humano, pero los docentes no deben olvidar que si no existe motivación, el escolar no optará por la lectura, sino por una de las posibilidades que le ofrece el espacio en que se desenvuelve.

A criterio de autora la actividad social que presupone promover la lectura solo se puede lograr con orientación planificada, dirigida desde un enfoque pedagógico y cultural. Lo cierto es que nunca estuvo demasiado claro qué papel debe desempeñar la institución escolar en los esfuerzos de promoción y animación por la lectura; y es que aquí hay uno de los problemas esenciales aún por definir o profundizar, no ya en Cuba sino en cualquier otro lugar.

En suma, una biblioteca escolar para que sea eficaz en la formación del lector debe caracterizarse por ser amena, racional y dinámica; debe lograrse el encuentro con los libros como actividad alegre y placentera y no como una acción obligatoria ante la cual los escolares no tienen otro remedio que aceptarlo.

La motivación es esencial para lograr altos niveles de desarrollo en la capacidad y en la actitud lectora de los escolares. Los motivos se consideran generadores de cambios en el entrenamiento del lector durante el proceso de su formación expresado en la posibilidad de adquirir conocimientos sobre lo que lee, desarrollar habilidades para el manejo de la información, posibilidad de escoger el material que desea leer, asumir comportamientos más adecuados desde su posición de usuario en una biblioteca u otro centro de información, practicar la lectura como un verdadero acto de placer; incrementar la confianza en sí mismo como lectores e incluso modelar sus patrones de conducta en sentido general (Fowler, 2000).

La motivación juega un papel fundamental para desarrollar el interés por la lectura; pues cuando esta tiene significación para el niño o el joven, se realiza la actividad de leer con mayor eficiencia, por ello se hace necesario para un promotor de lectura conocer y profundizar acerca de la motivación. Este término ha sido abordado en la literatura especializada por diferentes autores. 
Para González (1995; p. 19) la motivación es:

La compleja integración de procesos psíquicos que en su constante transformación y determinación recíproca con la actividad externa y sus objetivos y estímulos, va dirigida a satisfacer las necesidades del hombre y en consecuencia, regula la dirección (el objeto-meta) y la intensidad o activación del comportamiento, manifestándose como actividad motivada.

Definición que se asume en esta investigación al tener presente la propia dinámica humana, siempre vinculada a la satisfacción de sus necesidades con la activación del comportamiento del usuario. González (2000) precisa que los puntos de partida en el estudio de la motivación los encontramos en las necesidades y los motivos. González (2004) expresa: es una compleja integración de procesos psíquicos que regula la dirección (el objeto- meta) y la intensidad o activación del comportamiento.

Arias (2004, p. 82), refiere que la motivación es lo que se encuentra detrás de nuestro comportamiento, la razón de que llevemos a cabo lo que hacemos; es la fuerza que inicia, dirige y sostiene la conducta hacia una meta para así mantener una conducta determinada, dicha fuerza puede tener su origen en condiciones externas y estar dirigidas a satisfacer necesidades ajenas a la naturaleza del acto mismo.

A consideración de Rodríguez Pérez (2003, p. 85), la motivación es la fuerza motriz que mueve a una persona a tener un comportamiento determinado, una conducta concreta. Dicha fuerza puede tener su origen en condiciones externas y estar dirigida a satisfacer necesidades ajenas a la naturaleza del acto mismo, por ejemplo para obtener un premio o evitar un castigo. La motivación aparece como fuerza interior que motiva determinada actuación cuando la persona ha alcanzado cierta madurez.

Valorando los elementos abordados por estos autores, la autora considera que los motivos son elementos esenciales en la personalidad que orienta la actividad del individuo, se puede adicionar al respecto que la interacción media como elemento casi determinante en el desarrollo de las habilidades lectoras, declarando que la familia, la comunidad y los medios masivos de comunicación juegan un papel fundamental en este sentido.

Para la definición de este concepto se tiene en cuenta el término esfera motivacional, los motivos son impulsos para la acción vinculados con la satisfacción de determinadas necesidades y se diferencian entre sí por el tipo de necesidad al que responden y por el contenido concreto de la actividad en la cual ellos se manifiestan.

Es oportuno reconocer que en la motivación está presente la iniciativa asumida como elemento esencial para crear un producto original y la persistencia estrechamente 
vinculada con la independencia poniéndose de manifiesto el principio de la sistematización, sino se hace reiterada, casi intensa, la demostración de que la actividad lectora contribuye a cambios sustanciales en la personalidad.

Herrera (2000), ofrece consideraciones al respecto y plantea que se precisa entre otros elementos, la aplicación creativa de técnicas de animación que eleven el nivel de motivación de los escolares hacia la lectura, sin olvidarse nunca que la lectura debe constituir un placer, al cual se regresa porque se siente la honda y libre necesidad de repetirlo. A tales efectos es necesario abordar algunos elementos que sirven de sustento en la presente investigación.

La formación de buenos lectores, es una de las misiones cardinales e indelegables de la escuela, y en tal sentido esta devendrá el centro coordinador de las acciones e influencias que integrarán todo el quehacer pedagógico en función del logro de estos propósitos. A criterio de Herrera (2000), aunque cada docente debe encontrar su camino en función de sus realidades y experiencias, es recomendable la aplicación creativa de técnicas de animación que eleven el nivel de motivación hacia la lectura y que tomen en cuenta la trascendencia de elementos lúdicos en los comportamientos del escolar como lector. No puede olvidarse nunca que la lectura debe constituir un placer, al cual se regresa porque se siente la honda y libre necesidad de repetirlo.

Un punto de partida imprescindible lo constituye un concepto clave: la animación de la lectura. Áreas (2008), la concibe como un acto consciente realizado para producir un acercamiento afectivo e intelectual a un libro concreto, de forma que este contacto produzca una estimación genérica hacia los libros. Esta se inserta dentro de las acciones de promoción, pero se entiende directamente con los textos. Se denomina animación de la lectura a las actividades que tienen como objetivo animar el texto, darle ánima, es decir, dotar de vida a los libros, hacer que habiten en el conocimiento, la experiencia y la imaginación de los lectores. Es una actividad que requiere de un intermediario, conocedor tanto de los destinatarios como del material literario que se desea difundir entre ellos, la cual se desarrolla con el propósito de crear una relación afectiva entre el libro y un grupo de lectores, como punto de partida para establecer un vínculo permanente y sólido con los demás libros.

La citada autora, agrega además, que toda actividad de animación es una actividad de promoción, pero no viceversa. Es actuación intencional, que, con estrategias de carácter lúdico y creativo, transforman actitudes individuales y colectivas en torno a la lectura y el libro. Actividades participativas, interacción con metodología abierta y flexible adaptada a las personas para las que se proyectan. Procedimiento en el que se utilizan de forma intencional estímulos con el fin de transformar actitudes para lograr determinada conducta. Procedimiento de aprendizaje intencionalmente educativo, cuyo objetivo final será la autoeducación que acercará al sujeto al hábito lector. 


\section{EDUCACIÓN}

Considerando las precisiones que se realizan en torno a la animación de la lectura y que resultan fundamentales para proyectar el trabajo en este sentido, la autora se adscribe al concepto referido en párrafos anteriores.

La animación de la lectura propone:

1. Que el escolar no lector, descubra el libro.

2. Ayudarle a pasar de la lectura pasiva, a la lectura activa.

3. Desarrollar en él el placer de leer.

4. Ayudarle a descubrir la diversidad de los libros.

$\mathrm{Al}$ escolar hay que introducirlo en la literatura mediante una lectura para que:

1. Pueda comprender.

2. Le haga gozar.

3. Que le permita reflexionar.

Aspectos a tener en cuenta durante el acto de animación de la lectura:

1. Seguir con fidelidad las estrategias.

2. Que las animaciones se realicen con carácter lúdico, diferenciándolas de las de clases y alejándolas de todo aire didáctico.

3. Darle aire de celebraciones.

4. No pedirle a los escolares jamás que realicen un "trabajo" como consecuencia de su participación en una animación.

5. Puede hacerse en cualquier lugar dentro de la escuela o la comunidad.

Técnicas que se sugieren para la animación de la lectura:

1. Una lectura equivocada.

2. Están o no están.

3. La frase falsa.

4. Este es el título.

5. Antes o después.

6. Gazapos.

7. Combate amistoso.

8. Se habla de...

Áreas Leiva (2010), en su artículo "La escuela y la formación de lectores", sugiere otras técnicas de animación de lectura, entre las que se encuentran: 
1. Hoy vamos a conversar sobre...

2. Ahora te voy a leer...

3. Hagamos una campaña publicitaria.

4. Menú literario.

5. ¿Quién sabe más sobre "ese" autor?

6. El diario del lector.

7. ¿Cómo se hace un libro?

8. Lectura compartida.

9. Encuentra el libro a partir de "pistas".

10. ¿De qué trata esta historia? Ejercicios de anticipación.

En el texto Español 8, Hablemos sobre promoción y animación a la lectura, esta autora hace referencia además a:

1. Lectura "rompecabezas".

2. Cartas a fantasía.

3. El cuaderno viajero.

Todo lo apuntado en el capítulo nos lleva a considerar que en el panorama educativo y cultural, cada vez más saturado con textos sobre la promoción y la animación de la lectura para el acceso a la verdadera cultura y en especial al libro, la escuela ocupa un lugar prioritario, en pos de formar lectores que vean en cada ejemplar fuente de satisfacción y disfrute personal y que la biblioteca escolar juegue la acción creadora y recreadora en la ineludible tarea de hacer de la lectura una poderosa palanca de la plenitud humana.

Ese propósito requiere no sólo de voluntad y amor. Se necesita de la investigación científica, de la profundización en la bibliografía seria y actualizada y de la preparación de docentes y bibliotecarios. En todas estas direcciones se ha trabajado con creciente profesionalidad, pero aún quedan grandes retos por vencer.

\section{Metodología}

El estudio se desarrolló en el período comprendido de septiembre de 2018 a marzo de 2019, a partir de la aplicación del método de experimento pedagógico, en su modalidad de pre - experimento, con un diseño de pre - test y pos - test, con control de la variable dependiente: Nivel de motivación por la lectura. Este se expresa en el estilo de comportamiento de los escolares en relación con las necesidades e intereses por la lectura y las vivencias afectivas que experimentan como lectores en función de su satisfacción personal.

La población seleccionada la constituyen los 20 escolares que cursan el sexto grado en la escuela primaria José Martí del municipio La Sierpe, Cuba. No fue necesario determinar un criterio de selección muestral ya que la población posee características 


\section{EDUCACIÓN}

en cuanto a extensión y posibilidades de interacción con la investigadora que propician el trabajo con la totalidad de los sujetos.

Como rasgos que permitan la referencia empírica de la variable dependiente se determinan como indicadores los siguientes:

1. Evidencia placer personal durante el acto lector.

2. Solicitan libros para disfrute personal.

3. Visitan espontáneamente la biblioteca en su tiempo libre.

4. Participación en actividades extradocentes y extraescolares asociadas a la actividad de lectura.

Teniendo en cuenta los indicadores, se seleccionaron los métodos fundamentales para determinar el estado de la variable dependiente antes (pre - test) y después (post - test) de la introducción de la variable independiente. Los métodos utilizados en ambos momentos fueron: la prueba pedagógica (pre - test y pos - test), y el análisis de documentos.

El análisis de la situación con respecto a la motivación por la lectura de los escolares de sexto grado de la escuela "José Martî" del municipio La Sierpe, Cuba.Se realizó a partir de la aplicación de instrumentos que abarcaron la revisión de: Exigencias del Modelo de la escuela primaria para la dirección por el maestro de los procesos de educación, enseñanza y aprendizaje, Programa curricular para bibliotecas escolares, Normas relativas a la organización y funcionamiento de las unidades de información que conforman el Sistema de Información para la Educación y Estadísticos de asistencia y préstamo. Complementan la información recopilada entrevistas, encuestas y observación al desempeño de los escolares en este proceso.

El grupo de instrumentos aplicados permitió realizar una valoración de la situación que presenta la motivación por la lectura en los escolares de sexto grado de la escuela primaria “José Martí". Es evidente que en el tema que ocupa a esta investigación existen aún insuficiencias, corroborándose en el estilo de comportamiento de los escolares en relación con las necesidades e intereses por la lectura y las vivencias afectivas que experimentan como lectores en función de su satisfacción personal.

Durante el estudio inicial realizado, se confirma la necesidad de elaborar actividades de animación de la lectura dirigidas a elevar los niveles en la motivación por esta de los escolares antes mencionados. 


\section{Resultados y discusión}

El diagnóstico realizado corrobora la problemática existente en relación con la motivación por la lectura en los escolares de sexto grado de la escuela primaria "José Martî", en las tablas se muestran los resultados obtenidos durante la etapa de pre test:

Tabla 1: Resultados obtenidos antes de aplicada la propuesta (pre test)

\begin{tabular}{ccccccc}
\hline Indicador & Alto & $\%$ & Medio & $\%$ & Bajo & $\%$ \\
\hline 1.1 & 1 & 5 & 4 & 20 & 15 & 75 \\
1.2 & 1 & 5 & 3 & 15 & 16 & 80 \\
1.3 & 1 & 5 & 6 & 30 & 13 & 65 \\
1.4 & 1 & 5 & 4 & 20 & 15 & 75 \\
\hline
\end{tabular}

En correspondencia con el objetivo de esta investigación, se realiza una propuesta de actividades de animación de la lectura, sobre la base de los presupuestos teóricos que la sustentan.

Para la realización de las actividades se tuvo en cuenta además las características de los escolares de tercer momento del desarrollo del escolar primario, los que se inician en la etapa de la adolescencia. Al situarlos entre 10 y 12 años. En estas se considera como elemento indispensable en este momento del desarrollo las fases en la evolución de los interese literarios, tomando como base la agrupación por edades, con carácter solo indicativo y flexible, asumiendo las consideraciones al respecto (Herrera,2006): sin que se abandonen del todo las preferencias anteriores, se manifiesta una disposición hacia los relatos de orientación realista y los textos de divulgación científica, histórica, deportiva y cultural. Los niños comienzan a leer libros completos de cierta extensión, como novelas de aventuras u otras de acción o ambiente cautivadores.

En la investigación se asume como concepto de actividades de animación de la lectura como: "un acto consciente realizado para producir un acercamiento afectivo e intelectual a un libro concreto, de forma que este contacto produzca una estimación genérica hacia los libros, teniendo como objetivo, animar el texto, darle ánima, es decir, dotar de vida a los libros, hacer que habiten en el conocimiento, la experiencia, la imaginación de los lectores, la cual se desarrolla con el propósito de crear una relación afectiva entre un libro y un grupo de lectores, como punto de partida para establecer un vínculo más permanente y sólido con los demás libros; actuación intencional, que, con estrategias de carácter lúdico y creativo transforma actitudes individuales y colectivas en torno a la lectura y el libro" (Áreas, 2003, p. 58).

Las actividades de animación de la lectura dirigidas a la motivación por estas en los escolares de sexto grado de la escuela primaria que se proponen, se distinguen por las siguientes características: 


\section{EDUCACIÓN}

1. Un estilo participativo y democrático: se expresa en el reconocimiento de los escolares como agentes activos en la ejecución de las actividades de animación. Concebir esta como característica general de la propuesta favorece la creación de un ambiente psicológico favorable que minimice la resistencia que los escolares puedan ofrecer.

2. Se asume el estilo participativo y democrático como las oportunidades que tienen los sujetos para participar con independencia y conscientemente en las actividades, estará dado tanto por el nivel de implicación en la búsqueda del conocimiento y las exigencias de estas para adquirirlo y utilizarlo, así como por las propias exigencias que deberá propiciar un rico intercambio y comunicación de los sujetos entre sí, con un alto grado de responsabilidad y satisfacción, capaz de despertar vivencias positivas en los escolares; mientras, actúa sobre el componente volitivo, afectivo, intelectual, social al concederles oportunidades para que juntos propongan, tomen iniciativas, ejecuten y controlen determinadas acciones.

3. Se debe manifestar mediante el análisis de contradicciones, de errores, la selección de alternativas de solución a las actividades planteadas, deducciones, emisión de juicios, valoraciones, todo lo cual fomenta el compromiso con la actividad que se desarrolla y la utilización de mecanismos de autocontrol.

4. Empleo de técnicas de animación a la lectura: integradas por acciones concebidas con el objetivo de formar lectores, logrando que la lectura, además de una necesidad llegue a constituir un placer irrenunciable, estimular situaciones en una dinámica saludable y placentera, que aporten gustos, preferencias y formen hábitos. Relacionar la lectura con momentos de placer, participación e intercambio llevan al escolar al contacto directo con el material de lectura, con el maestro como mediador, animando el texto, es decir, dotar de vida a los libros, hacer que habiten en el conocimiento, la experiencia y la imaginación, en función de establecer un vínculo más permanente y sólido. El carácter lúdico y creativo de las técnicas transforma actitudes individuales y colectivas en torno a la lectura y el libro con una metodología abierta y flexible, adaptada a los escolares para los que se proyectan. Estas técnicas pueden ser previas a la lectura, posteriores o realizadas durante la lectura. Se asumen en la investigación las técnicas que sugiere Áreas (2010).

5. Provocar efectos emotivos que hagan vivir al escolar la acción: uno de los retos de la didáctica es rescatar a la clase de lectura como un espacio y un momento de placer para los escolares, de manera tal que fomente el gusto por leer, la sensibilidad por los libros y la creatividad. Para ello, se necesita desencadenar mecanismos que permitan el descubrimiento lúdico, el intercambio de impresiones y percepciones sobre el texto, el contagio con las estructuras lingüísticas, 
la toma de conciencia de la lectura del texto, la vinculación con las experiencias personales, familiares y comunitarias; el goce estético y la audacia imaginativa. De lo contrario, pueden dominarse bien las habilidades, pero sin pericias en el proceso lector ni capacidad para sentir y vivir la lectura.

Para darle vitalidad a las actividades se debe desentrañar en los escolares los sentimientos que lo comprometen con el contenido del texto para que se sientan parte de él; inducirlos a que capten sus esencias y las variadas proyecciones de sus significados ocultos, asuman ideas que le conceden valor a sus vidas, se identifiquen con las aventuras y desventuras de los personajes, infieran sentimientos, modos de actuación, anticipen sucesos, evoquen imágenes, agreguen otros elementos y conquisten nuevos horizontes culturales.

Cada uno de las actividades de animación de la lectura se estructuraron del siguiente modo: título, objetivo, grado, tiempo, metodología y bibliografía. Estos aspectos fueron emitidos en el documento "Normas relativas a la organización y funcionamiento de las unidades de información que conforman el Sistema de Información para la Educación", las cuales se asumen en este trabajo. Se considera, además, lo planteado por Áreas Leiva, (2010, p. 47), al referirse a este tipo de actividad, "Actividades participativas, interacción con metodología abierta y flexible adaptada a las personas para las que se proyecta".

A manera de síntesis puede afirmarse que de modo general las actividades que se proponen contribuyen a la motivación por la lectura en los escolares de sexto grado de la escuela primaria, ya que en todos los indicadores evaluados se observaron transformaciones de tendencia positiva, tal como se ilustra en los gráficos y tablas que siguen:

Tabla 2: Resultados cuantitativos obtenidos en relación con el comportamiento de los indicadores, antes (pre test) y después (pos test) de la introducción de la variable independiente.

\begin{tabular}{|c|c|c|c|c|c|c|}
\hline Indicador & Alto & Medio & Bajo & Alto & Medio & Bajo \\
\hline 1.1 & 5 & 20 & 75 & 70 & 30 & 0 \\
\hline 1.2 & 5 & 15 & 80 & 85 & 15 & 0 \\
\hline 1.3 & 5 & 30 & 65 & 80 & 20 & 0 \\
\hline 1.4 & 5 & 20 & 75 & 75 & 25 & 0 \\
\hline
\end{tabular}




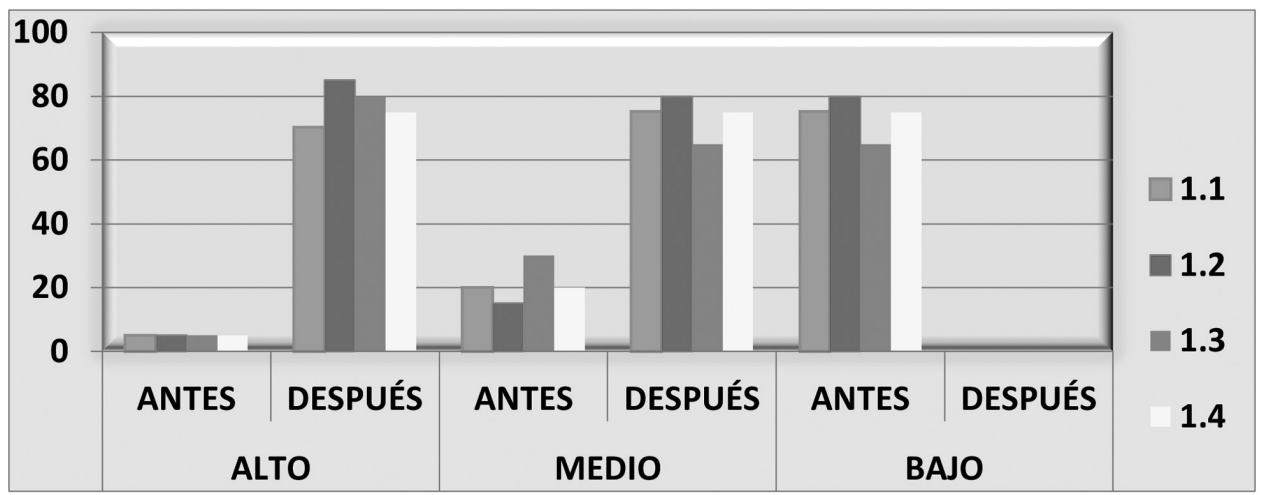

Gráfico No. 1: Análisis comparativo de los resultados antes y después de aplicada la propuesta.

\section{Estas tendencias positivas se evidencian en:}

El nivel de motivación por la lectura que se traduce en el placer personal evidente durante el acto lector; solicitan libros para disfrute personal, ven en ellos el mejor modo de emplear su tiempo libre; visitan de forma espontánea la biblioteca, permaneciendo gran parte de su tiempo libre en ella y participan de forma animada en actividades extradocentes y extraescolares asociadas a la lectura, contagiando con facilidad a los demás.

\section{Conclusiones}

1. La revisión bibliográfica realizada permite afirmar que, en el proceso de formación de lectores, la biblioteca escolar representa un eslabón esencial en la creación y consolidación del hábito de leer en los escolares de la escuela primaria, el cual debe recibir en este espacio el impulso necesario en pos de la motivación por la lectura, esto es posible lograr a partir del empleo de métodos y procedimientos adecuados.

2. La constatación inicial realizada permitió corroborar que aún persisten insuficiencias en la escuela primaria en cuanto a la motivación por la lectura, en este sentido se aprecia predisposición durante el acto lector, no asocian la lectura como un acto placentero y un limitado número de escolares practican la lectura recreativa en el tiempo libre.

3. Como propuesta de solución al problema científico declarado se elaboraron actividades de animación de la lectura con el propósito de motivar la lectura en los escolares de sexto grado, estas se caracterizan por un estilo participativo y democrático, el empleo de técnicas de animación a la lectura y provocan efectos emotivos que hagan vivir al escolar la acción.

4. La validación de las actividades animación de la lectura mediante la aplicación del pre- experimento evidencian la evolución favorable en los indicadores declarados, vertido en el placer personal evidente durante el acto lector; solicitan 
libros para disfrute personal; visitan espontáneamente la biblioteca y participan de forma animada en actividades extradocentes y extraescolares asociadas a la lectura; confirmando la validez y pertinencia de la propuesta.

\section{Recomendaciones}

1. Orientar nuevos estudios para la motivación de la lectura en otros grados de la enseñanza primaria.

2. Divulgar los resultados de la investigación entre los funcionarios encargados de la preparación didáctica metodológica de los bibliotecarios del territorio a fin de que puedan perfeccionar su desempeño profesional.

3. Generalizar la propuesta de actividades para la motivación de la lectura a otros centros del territorio.

\section{Agradecimiento}

Esta publicación obtuvo el financiamiento de: El Fondo de Asistencia Internacional de los Estudiantes y Académicos Noruegos, (SAIH).

\section{Lista de referencias}

Arias Leiva, G. (2003) Leer antes de leer. La Habana: Editorial Pueblo y Educación.

Áreas Leiva, G. (2008). Hablemos sobre promoción y animación a la lectura. Español 8. La Habana: Editorial Pueblo y Educación.

Arias Leiva, G. (2010) Horadar en el texto. La Habana: Editorial Pueblo y Educación.

Fowler Calzada, V. (200o). La lectura, ese poliedro. La Habana: Editorial Biblioteca Nacional José Martí.

García González, D. (2011). Actividades de animación que contribuyen a la motivación por la lectura. (Tesis de Maestría). ISP “Capitán Silverio Blanco Núñez, Sancti Spíritus.

González Serra, D. (2000). La motivación. Una orientación para su estudio. La Habana: Editorial Científico- Técnica.

Henríquez Ureña, C. (1975). Invitación a la lectura. La Habana: Editorial Pueblo y Educación.

Herrera Rojas, R. L. (2009). Magia de la letra viva. Cómo formar lectores en la escuela. Sancti Spiritus: Editorial Luminaria.

Rodríguez Pérez, L. (2003). Español para todos. La Habana. Editorial Pueblo y Educación. 